\title{
Large indoor cage study of the suppression of stable Aedes aegypti populations by the release of thiotepa-sterilised males
}

\author{
René Gato ${ }^{1}{ }^{+}$, Rosemary Susan Lees ${ }^{2}$, Rosa Y Bruzon ${ }^{1}$, Ariamys Companioni ${ }^{1}$, \\ Zulema Menendez ${ }^{1}$, Aileen González ${ }^{1}$, Misladys Rodríguez ${ }^{1}$ \\ ${ }^{1}$ Institute of Tropical Medicine Pedro Kourí, La Habana, Havana, Cuba \\ ${ }^{2}$ Polo d'Innovazione Genomica, Genetica e Biologia, Polo Unico di Medicina Santa Maria della Misericordia, Perugia, Italy
}

The sterile insect technique (SIT) is a promising pest control method in terms of efficacy and environmental compatibility. In this study, we determined the efficacy of thiotepa-sterilised males in reducing the target Aedes aegypti populations. Treated male pupae were released weekly into large laboratory cages at a constant ratio of either 5:1 or 2:1 sterile-to-fertile males. A two-to-one release ratio reduced the hatch rate of eggs laid in the cage by approximately a third and reduced the adult catch rate by approximately a quarter, but a 5:1 release drove the population to elimination after 15 weeks of release. These results indicate that thiotepa exposure is an effective means of sterilising Ae. aegypti and males thus treated are able to reduce the reproductive capacity of a stable population under laboratory conditions. Further testing of the method in semi-field enclosures is required to evaluate the mating competitiveness of sterile males when exposed to natural environmental conditions. If proven effective, SIT using thiotepa-sterilised males may be incorporated into an integrated programme of vector control to combat dengue in Cuba.

Key words: Aedes aegypti - chemosterilisation - thiotepa - sterile insect technique

Since 1981, when the first haemorrhagic dengue epidemic in the Americas was reported in Cuba, the country has remained dengue free, with the exception of sporadic, isolated clusters of cases (Guzman 2012). Aedes aegypti control programmes in Cuba rely on the application of the organophosphate temephos for larval control and the application of pyrethroids as adulticides. However, the widespread use of insecticides since 1981 has led to the development of resistance (Bisset et al. 2011).

The sterile insect technique (SIT) (Knipling et al. 1968) is a remarkably promising vector control method in terms of efficacy, efficiency and environmental compatibility. In classical SIT, the sterility of insects is induced by exposure to radiation or chemical mutagens (Benedict \& Robinson 2003).

Several alkylating agents and aziridinyl compounds have been tested as mosquito sterilants (Dame et al. 2009). Thiotepa is an alkylating agent developed in the 1950s that is being used in many chemotherapy regimens for the treatment of human solid tumours (van der Wall et al. 1995). The exposure of Ae. aegypti to thiotepa induces irreversible sterility (White 1966).

There are concerns over the use of alkylating agents, including the safety of those handling the chemicals during sterilisation and the potential for residues remaining on released adults that could affect the environment

doi: 10.1590/0074-0276130519

Financial support: IAEA (CRP G34002)

+ Corresponding author: rene@ipk.sld.cu

Received 30 October 2013

Accepted 17 March 2014
(Borkovec 1976). The risk of environmental contamination with the sterilising agent from the release of mosquitoes exposed in the pupal stage is very low because the amount of the thiotepa residue detected in adults is negligible (La Brecque et al. 1972, Seawright et al. 1973, 1976). Furthermore, the common use of thiotepa in cancer therapy has led to the development of suitable procedures for the safe disposal of waste (Barek et al. 1998). The success of SIT in controlling the malaria vector Anopheles albimanus in El Salvador demonstrated the effectiveness of thiotepa as a sterilant (Lofgren et al. 1974), so its potential as a sterilising agent for SIT programmes should not be overlooked. The availability of an alternative method of sterilisation is particularly important because the purchase and maintenance of irradiators is becoming increasingly difficult due to security fears, especially in developing countries (Mehta \& Parker 2011).

The performance of sterile mosquitoes following release is essential for the success of SIT (Dame et al. 2009). Somatic damage as a consequence of the sterilisation process has been well documented (Proverbs 1969) and is known to affect the longevity (Oliva et al. 2013), sexual vigour and competitiveness of sterilised male mosquitoes (Helinski \& Knols 2009), with severity depending on the species (Helinski et al. 2009).

Chemosterilants offer high levels of sterility and more competitive insects compared with irradiation (El Gazzar \& Dame 1983, Helinski et al. 2009). Recently, we have shown that Ae. aegypti thiotepa-treated males could effectively reduce the reproductive capability of a target insect's population under laboratory conditions (Gato et al. 2013).

The objective of this study was to determine the efficacy of releasing thiotepa-treated males at a 2:1 or 5:1 sterile:fertile male ratio in reducing the stable target $A e$. aegypti populations, measured through the egg hatch 
and adult catch rate in large indoor cages. Thiotepa was selected as the sterilant for three major reasons. First, the common use of thiotepa in the treatment of human cancer has led to the development of safe procedures for handling the chemical and waste disposal (Barek et al. 1998). Second, the treatment of Ae. aegypti with thiote$\mathrm{pa}$ at the pupal stage ensures complete and irreversible sterility (White 1966). Third, the amount of thiotepa in adults is insignificant when the mosquitoes are treated at the pupal stage (Seawright et al. 1973, 1976). We discuss the results in relation to the potential for the application of this technique as a part of an integrated programme for vector management in Cuba.

\section{MATERIALS AND METHODS}

Mosquitoes - A wild-type strain of Ae. aegypti was used. To obtain a high genetic diversity, the strain was derived from a mixture of immature mosquitoes collected in five municipalities of Havana, Cuba. The adults were reared in $18 \times 18 \times 18$ inch collapsible cages (BioQuip, USA) maintained at $26^{\circ} \mathrm{C} \pm 0.5^{\circ} \mathrm{C}$ in $80-85 \%$ relative humidity (RH) with a photoperiod of 12:12 h (light/dark). A continuous supply of sucrose solution was provided. Female mosquitoes were given access to an anesthetised mouse and allowed to blood feed for 30 min weekly. The larvae were fed finely powdered fish food.

Sex separation was based on pupal size; Ae. aegypti female pupae are visibly larger than male pupae and hatch later (Forattini 2002). Pupae were individually removed from the rearing tray using a disposable pipette. In all cases, the mosquitoes were sexed prior to transferring them to the large cages.

Sterilisation - A $0.6 \%$ solution of thiotepa (Sigma, Belgium) was prepared in distilled water buffered to $\mathrm{pH}$ 8.6. Young male pupae ( $1-4 \mathrm{~h}$ old) were immersed in this solution for $6 \mathrm{~h}$, rinsed twice in dechlorinated water and transferred to a Mosquito Breeder (BioQuip) for emergence. Because both fertile and sterile males were needed for each 'release' in the suppression phase, a fraction of the male pupae was treated with thiotepa.

Large cages - Three identical indoor screened cages ( $1.46 \mathrm{~m}$ in width, $1.85 \mathrm{~m}$ in length and $2.02 \mathrm{~m}$ in height) were used for the suppression trial. The exterior walls, floor and ceiling were made of concrete and room dividers between cages were a fine metallic mesh in appropriate aluminium frames (Fig. 1). Resting and hiding sites were provided in the form of the following household objects: cotton curtains, a chair, a table, a vase with artificial flowers and a shelf. A constant environment of $26^{\circ} \mathrm{C} \pm 2^{\circ} \mathrm{C}, 80-85 \% \mathrm{RH}$ and a $12: 12 \mathrm{~h}$ light:dark photoperiod was maintained.

Two treatment cages and one control cage were established as follows: cage A contained fertile females and fertile males at a 1:1 ratio (control); cage B contained fertile females, fertile males and sterilised males at a 1:1:5 ratio and cage $\mathrm{C}$ contained fertile females, fertile males and sterilised males at a 1:1:2 ratio.

Access to each cage was obtained through a sleeve opening through which a table could be reached where the oviposition containers, animals for blood-feeding and a constant sugar source were placed. Battery-operated CDC light traps were hung inside the cage and accessed through sleeves. Cages were randomly assigned to each treatment once a stable population had been established in all cages (the end of the pre-treatment phase).

Target populations (pre-treatment phase) - Stable mosquito colonies were established in each of the three cages during a 20 week pre-treatment phase. Seventy pupae of each sex were added for an initial population of 140 individuals per cage and 50 individuals of each sex per cage were added weekly.

The required number of each sex were added to a Mosquito Breeder (BioQuip) placed on the tables inside the cages and the devices were uncovered once daily to allow the emerged adult mosquitoes to escape.

Four restrained guinea pigs were placed on the table of each cage around sunrise for $30 \mathrm{~min}$ on alternate days as a blood-feeding source and mating stimulus. Continuous access was allowed to a sucrose source by placing sugar-water-soaked cotton pads in the cages.

Three oviposition containers consisting of glass trays (18 $\mathrm{cm}$ in diameter, $5 \mathrm{~cm}$ in height) lined with filter paper and filled with $900 \mathrm{~mL}$ of tap water were added to each cage. Females began to oviposit at week 4 . Water and filter papers were replaced to collect eggs three times per week (Monday, Wednesday and Friday) to prevent the eggs from hatching inside the cages. The eggs were counted once a week to determine the total egg lay per week. The eggs were counted under a dissecting microscope and the total oviposition was recorded by week. The eggs were allowed to mature on the moist paper strips for seven days and were then immersed in tap water at $36^{\circ} \mathrm{C}$. The resulting larvae were fed to pupation.

The hatch rate was calculated as described by Benedict (2007). The filter papers with eggs were recovered from the hatching trays and were examined under a ste-

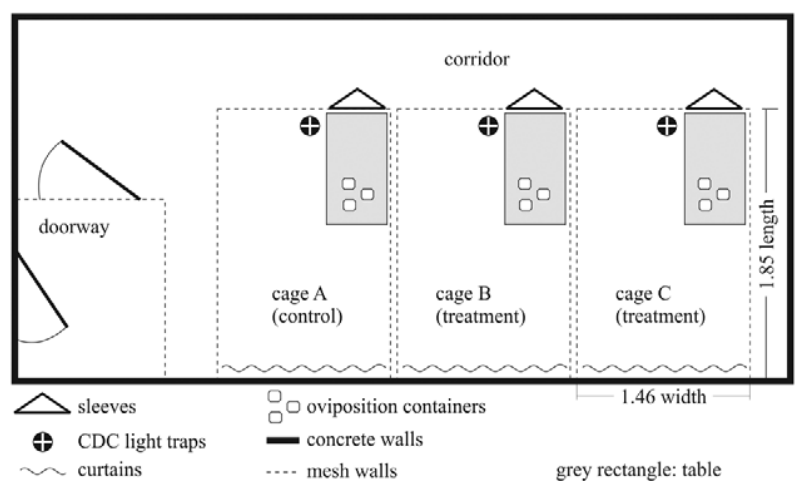

Fig. 1: sketch of large laboratory cages. Three identical screened cages just over $5 \mathrm{~m}^{3}$ were used. Cages were randomly assigned as follows: cage A contained fertile females and fertile males in a 1:1 ratio (control), cage B fertile females, fertile males and sterilised males in a 1:1:5 ratio and cage $\mathrm{C}$ fertile females, fertile males and sterilised males in a 1:1:2 ratio. The access to each cage was achieved through of sleeves. A table was located inside the cages to place animals for mosquito blood-feeding, oviposition containers, recipients with pupae to release and sugar source. CDC light traps were hung inside the cage and again accessed through sleeves. 
reoscope. Typically, the operculum of hatched eggs are somewhat dislodged. The hatch rate was assessed by the following formula: (number of hatched eggs/total number of eggs) x 100 .

The adult populations were monitored weekly from week 2 using CDC light traps turned on at 08:00 am every Monday; at this time, the rooms were kept in the dark for $10 \mathrm{~min}$. Thereafter, the room light was turned on and the traps were quickly removed from the cages, while being kept operational to prevent mosquitoes escaping. The collected mosquitoes were gently removed by a manual aspirator, counted by sex and returned to their respective cages. The adult mortality rate associated with the trapping and manipulation was $2.5 \%$.

The end of the pre-treatment phase was determined by the observation of a stable baseline population for three consecutive weeks in all cages.

Suppression phase - Throughout the suppression phase, pupae were added to each cage each week to maintain the population in addition to the sterile males that were added to the treatment cages to simulate releases in an SIT programme. Each week, 50 male and 50 female pupae were added to the control cage. The number of pupae added to each treatment cage during the suppression phase was adjusted to reflect the impact of the thiotepa-treated males on the egg quantity and fertility. The percentage of female pupae added back to the control cage was calculated from the number of fertile eggs laid (total number of eggs laid multiplied by hatch rate). For example, 6,254 eggs were laid in the control cage (A) in week 25 , of which $64.3 \%$ hatched, yielding 3,939 fertile eggs laid in total; thus, the 50 female pupae represented $1.26 \%$ of the total fertile eggs laid. Therefore, $1.26 \%$ of the fertile eggs collected from each of the treatment cages were added back that week in the form of female pupae and the same number of fertile male pupae, rounded down, were also added. Then, a sufficient number of sterile (thiotepa-treated) male pupae was added back to achieve a 5:1 and 2:1 ratio (sterile male:fertile male) in treatment cages B and C, respectively. For example, on week 25,20 female pupae were added to cage
B (1.26\% of the 1,633 fertile eggs collected from the cage that week) and therefore, 100 sterile males and 20 fertile males were also added. Thus, the "release ratio" (sterile males released:existing male population) in the treatment cages remained constant throughout the suppression phase and the relative population sizes were maintained by the addition of female and fertile male pupae as a proportion of the number of fertile eggs collected.

Ethics - This study was carried out according to the principles expressed in the Declaration of Helsinki. The protocols were approved by the Institutional Research Ethical Committee at the Institute of Tropical Medicine Pedro Kourí (certificate \#54966). The permission for the use of guinea pigs was obtained from the institutional ethical committee after demonstrating that all measures were taken to avoid unnecessary suffering and severe damage to the animals.

\section{RESULTS}

This study aimed to determine the efficacy of thiotepa-treated males to eradicate stable cage populations of Ae. aegypti when introduced into these populations at the pupal stage at 5:1 and 2:1 ratios relative to the fertile male populations. Target populations were established in three identical laboratory cages just over $5 \mathrm{~m}^{3}$ in volume by adding 50 male and 50 female pupae weekly to an initial population of 140 individuals. After an initial drop in the hatch rate between weeks 4 (the 1st week of oviposition) and 7 , the hatch rate stabilised at approximately $65-70 \%$ and remained stable with only minor fluctuations until the suppression phase was initiated at week 20 (Fig. 2). The weekly adult catch rate (number of adult females caught in a 10 min trapping session) rose rapidly from approximately five per cage in week 2 to approximately 20 per cage by week 8 , after which time it stabilised in all cages (Fig. 3). The total egg production and eggs laid per female per week followed the same pattern as the egg hatch rate (data not shown). One of these stable populations was randomly assigned as a control cage and the others were used as treatment cages with two or five sterile male pupae added weekly for each fertile male pupa.

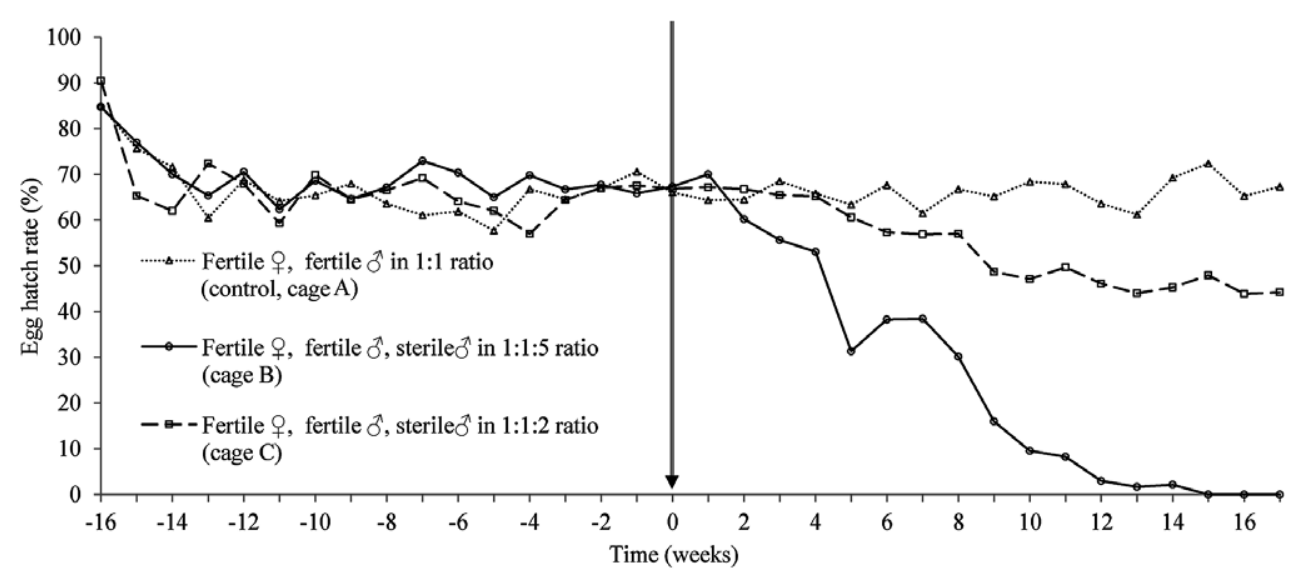

Fig. 2: hatch rate of Aedes aegypti in treatment and control cages. Stable hatch rate was achieved in each of the three cages during a pre-treatment phase. Vertical arrow represents the start of the release of thiotepa-treated mosquitoes into the cages B and C. 
In the control cage, the weekly introductions of 50 males and 50 females continued throughout the experiment and the reproductive parameters remained stable throughout the experiment. In the cage in which two sterile male pupae were introduced for every fertile male pupae reintroduced into the cage, which were set at a proportion equal to the proportion of fertile eggs added to the control cage each week, the hatch rate declined gradually for the first 10 weeks after the start of the suppression phase (Fig. 2). After this time, the hatch rate stabilised at approximately $45 \%$, compared to a rate of approximately $65 \%$ in the control cage. The adult catch rate did not respond to the releases until approximately five weeks into the suppression phase, after which time there was a small reduction in the adult catches in the 2:1 release cage, which stabilised at approximately 15 adults caught per trapping session compared to approximately 20 in the control cage (Fig. 3).

When five sterile males were added for each fertile male, however, the effect of sterile "releases" on the cage population was more marked. After only two weeks of suppression, the hatch rate had already begun to fall noticeably and by the fifth week of release, the egg hatch rate was reduced by a third (Fig. 2). This rapid decline continued until 15 weeks after the first release when no eggs collected from this cage hatched; three consecutive weeks of a $0 \%$ hatch rate were recorded before the experiment was stopped as elimination was deemed to have been achieved. The effect on the adult catch rate again took longer, but by 15 weeks into the suppression phase, the adult catch had fallen to only one adult per trapping session in the 5:1 release cage (Fig. 3), reaching and remaining at zero after 13 weeks of suppression.

The eggs laid per female per week remained fairly stable at 15-20 throughout the suppression phase. Some fluctuation in the 5:1 release cage (data not shown), likely caused by the very low number of eggs collected towards the end of the experiment. Total egg production followed a similar pattern as hatch rate and adult catch rate in the treatment cages, though never reached zero (data not shown). Population eradication was thus achieved with a $5: 1$, but not a 2:1 release of thiotepa-treated males.

\section{DISCUSSION}

The weekly release of thiotepa-sterilised males at a 5:1 ratio with the fertile males was shown to be sufficient to eradicate a large cage population of Ae. aegypti in 15 weeks, whereas eradication was not achieved with a 2:1 release ratio. The introduction of thiotepa-treated males continued until the 5:1 release ratio treatment cage had produced no fertile eggs for three weeks, at which time the experiment was ended. When the experiment was terminated, the hatch rate in the cage where sterile males were released at a 2:1 ratio exceeded $40 \%$ and the egg hatch and adult population (measured by adult catch during weekly trapping sessions) appeared to have stabilised by 10 weeks after the start of the suppression phase.

In applying the SIT to a target area, efficiency could be maximised by following the procedure described in this experiment and monitoring the hatch rate of eggs collected in the target area while releasing sterile males in a constant ratio to the wild male population. In this way, fewer males would need to be reared and released over time as the wild population is reduced. This might be desirable because the rearing capacity could then be used to target a second site for population suppression. Alternatively, the programme could release a constant number of sterile males so that as the programme progressed and the wild male population fell, the release ratio and thus the effectiveness of the releases would increase. This approach might be particularly suitable in an area where the immigration of mosquitoes from outside the target area is expected to be a problem, although the costs would be greater.

We have shown that the sterilisation of males with thiotepa and the release of these sterile pupae is an effective strategy for suppressing Ae. aegypti populations and can cause elimination of a target population in 15 weeks at a release ratio of 5:1; this length of time and scale of release is feasible in the setting of an SIT programme. Although further replicates would be desirable, this was not possible due to practical limitations and the results of this experiment are sufficiently reassuring regarding the performance of thiotepa-treated males to warrant moving directly to larger, more naturalistic suppression tri-

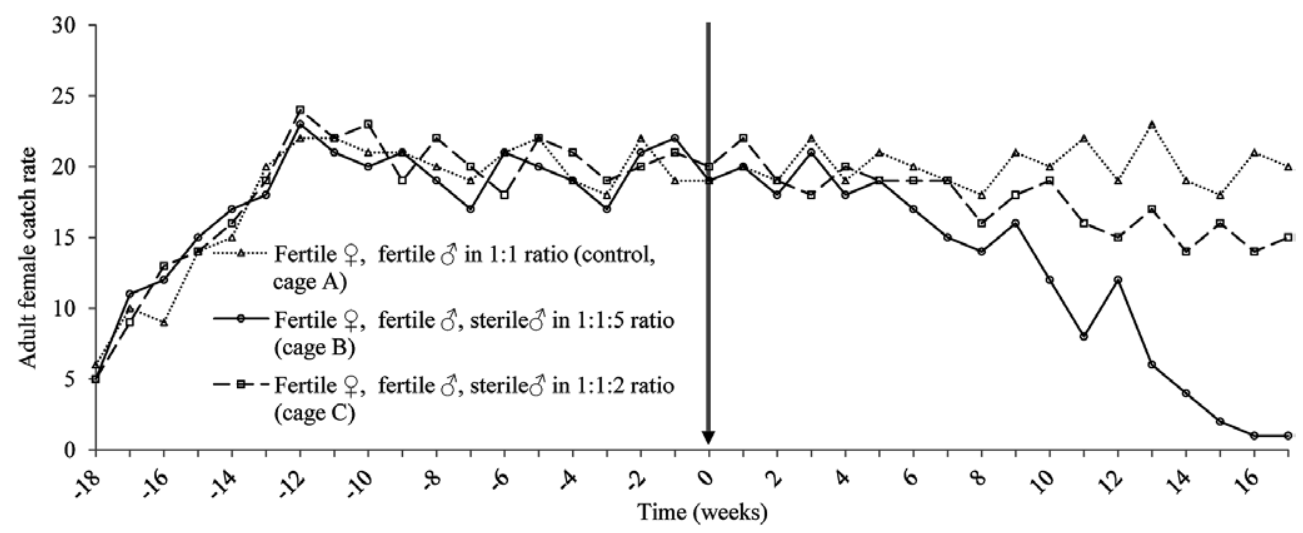

Fig. 3: female catch rate. Number of adult females caught weekly in a 10 min trapping session with CDC light traps. Stable mosquito colonies were established in each of the three cages during a pre-treatment phase. Vertical arrow represents the start of the release of thiotepa-treated mosquitoes into the cages B and C. 
als. There are, however, some limitations in laboratory experiments of this kind, even in large cages that have been modified to be similar to conditions found within a human dwelling where Ae. aegypti might be found resting given their propensity for using breeding sites close to human habitation in Cuba (Diéguez et al. 2011).

In a natural setting, the immigration of fertile insects and the emergence of fertile adults from breeding sites within the target area would make elimination more challenging; the immigration of fertilised females from outside the target site has been implicated in the failure of Culex quinquefasciatus and Ae. aegypti elimination using chemosterilised males in New Delhi in 1971 (Dame et al. 2009). The effect of adult immigration could be minimised by releasing sterile males into a buffer zone around the target suppression site or by successively controlling the mosquito population in zones in a sweeping pattern of releases (Dame et al. 2009). The effect of adults emerging from existing eggs laid in the trial site, a particular problem in Aedes spp whose eggs are resistant to desiccation, could be minimised by breeding site reduction prior to release.

There is the possibility that the competitiveness of the sterilised males could be impaired relative to that measured in laboratory trials by the less controlled and most likely harsher conditions found in the open field. In one recent example of such an effect, a transgenic line of Ae. aegypti was shown to be able to suppress stable laboratory populations through weekly releases of effectively sterile males within 10-20 weeks (de Valdez et al. 2011), but this same strain failed to achieve elimination in large outdoor field cages in southern Mexico with 17 weeks of releases (Facchinelli et al. 2013).

Finally, the density of adult mosquitoes in the cages at the end of the trial was unrealistically high compared to the population densities that are likely to be found in nature. For example, a study in Guasave Sinaloa, Mexico, measured an adult density index of approximately three Ae. aegypti adults per premises (García et al. 2011) and work in a Vietnamese village found a female adult abundance of between one-43 individuals per house (Jeffery et al. 2009). However, it is not practicable to maintain a stable population at these low densities in artificial conditions and a larger cage population allows for a more effective demonstration of the effectiveness of sterile "releases".

However, these results are valuable because they show that SIT using thiotepa sterilisation is a promising technique and that further exploration of the technique and testing on a larger and more natural scale would be worthwhile. Any gross reduction in the performance of sterilised males would likely have become apparent during the course of this trial, making elimination at a 5:1 ratio impossible. A five-to-one release ratio is relatively low, which is a promising sign for the success of an SIT programme using thiotepa sterilisation. The sterile males seem to have successfully competed for mates with the untreated males, as evidenced by the increased number of sterile eggs laid in the treatment cages relative to the control over time. Any impact on male performance caused by the mutagenic action of thiotepa is expected to be fairly minimal and if a modest reduction in competitiveness becomes apparent in more stringent testing, this could be overcome with an increased release ratio.

The present study is a significant step in addressing the gaps in the knowledge related to the inclusion of SIT as a practical part of integrated vector management for dengue control. Field trials to assess the effectiveness of the technology for application in Cuba will require the establishment of public confidence and the gathering of extensive resources and labour. However, although additional studies are required to guarantee the safety and efficacy of thiotepa as a sterilising method for mosquito releases, the success of the sterile mosquitoes in suppressing target cage populations in this study is likely to help gain acceptance for this method and other technologies based on SIT.

\section{ACKNOWLEDGEMENTS}

To Luke Alphey, for reviewing the paper.

\section{REFERENCES}

Barek J, Cvacka J, de Meo M, Laget M, Michelon J, Castegnaro M 1998. Chemical degradation of wastes of antineoplastic agents amsacrine, azathioprine, asparaginase and thiotepa. Ann Оссир Hyg 42: 259-266.

Benedict MQ 2007. Methods in Anopheles research - MR4. Determining egg hatch rates. Available from: mr4.org/Portals/3/MR4_Publications/Anopheles $\% 20$ Protocol $\% 20$ Manual $\% 20$ Second $\% 20$ Ed $\% 20$ v2011/2011\%20Complete\%20Manual\%20PDF\%20TOC.pdf.

Benedict MQ, Robinson AS 2003. The first releases of transgenic mosquitoes: an argument for the sterile insect technique. Trends Parasitol 19: 349-355.

Bisset JA, Rodriguez MM, Ricardo Y, Ranson H, Perez O, Moya M, Vazquez A 2011. Temephos resistance and esterase activity in the mosquito Aedes aegypti in Havana, Cuba increased dramatically between 2006 and 2008. Med Vet Entomol 25: 233-239.

Borkovec AB 1976. Control and management of insect populations by chemosterilants. Environ Health Perspect 14: 103-107.

Dame DA, Curtis CF, Benedict MQ, Robinson AS, Knols BGJ 2009. Historical applications of induced sterilisation in field populations of mosquitoes. Malar J 8 (Suppl. 2): S2.

de Valdez MR, Nimmo D, Betza J, Gong H, James AA, Alphey L, Black IV WC 2011. Genetic elimination of dengue vector mosquitoes. Proc Natl Acad Sci USA 108: 4772-4775.

Diéguez L, Cabrera SM, Prada Y, González E, Rodríguez R 2011. Bioecological studies of Aedes (St) aegypti in an urban area with low vector density in Camagüey province. Rev Cubana Med Trop 63: 64-69.

El Gazzar LM, Dame DA 1983. Effects of combinations of irradiation and chemosterilization on mating competitiveness of Culex quinquefasciatus Say. J Econ Entomol 76: 1331-1334.

Facchinelli L, Valerio L, Ramsey JM, Gould F, Walsh RK, Bond G, Robert MA, Lloyd AL, James AA, Alphey L, Scott TW 2013. Field cage studies and progressive evaluation of genetically-engineered mosquitoes. PLoS Negl Trop Dis 7: e2001.

Forattini OP 2002. Culicidologia médica: identificação, biologia, epidemiologia, Vol. 2, EDUSP, São Paulo, 864 pp.

García C, García L, Espinosa-Carreón L, Ley C 2011. Abundance and distribution of Aedes aegypti (Diptera: Culicidae) and dengue dispersion in Guasave Sinaloa, Mexico. Rev Biol Trop 59: 1609-1619. 
Gato R, Companioni A, Bruzón R, Menéndez Z, González A, Rodríguez M 2013. Release of thiotepa sterilized males into caged populations of Aedes aegypti: life table analysis. Acta Trop 132 (Suppl.): S164-S169.

Guzman MG 2012. Thirty years after the Cuban haemorrhagic dengue epidemic of 1981. MEDICC Rev 14: 46-51.

Helinski ME, Knols BG 2009. The influence of late-stage pupal irradiation and increased irradiated: un-irradiated male ratio on mating competitiveness of the malaria mosquito Anopheles arabiensis Patton. Bull Entomol Res 99: 317-322.

Helinski ME, Parker AG, Knols BG 2009. Radiation biology of mosquitoes. Malar J 8 (Suppl. 2): S6.

Jeffery JA, Yen NT, Nam VS, Nghia T, Hoffmann AA, Kay BH, Ryan PA 2009. Characterizing the Aedes aegypti population in a Vietnamese village in preparation for a Wolbachia-based mosquito control strategy to eliminate dengue. PLoS Negl Trop Dis 3: e552.

Knipling EF, Laven H, Craig GB, Pal R, Kitzmiller JB, Smith CN, Brown AW 1968. Genetic control of insects of public health importance. Bull World Health Organ 38: 421-438.

La Brecque GC, Bowman MC, Patterson RS, Seawright JA 1972. Persistence of thiotepa and tepa in pupae and adults of Culex pipiens fatigans Wiedemann. Bull World Health Organ 47: 675-676.

Lofgren CS, Dame DA, Breeland SG, Weidhaas DE, Jeffery G, Kaiser
R, Ford HR, Boston MD, Baldwin KF 1974. Release of chemosterilized males for the control of Anopheles albimanus in El Salvador. 3. Field methods and population control. Am J Trop Med Hyg 23: 288-297.

Mehta K, Parker A 2011. Characterization and dosimetry of a practical X-ray alternative to self-shielded gamma irradiators. Radiat Phys Chem Oxf Engl 80: 107-113.

Oliva CF, Maier MJ, Gilles J, Jacquet M, Lemperiere G, Quilici S, Vreysen MJ, Schooneman F, Chadee DD, Boyer S 2013. Effects of irradiation, presence of females and sugar supply on the longevity of sterile males Aedes albopictus (Skuse) under semi-field conditions on Reunion Island. Acta Trop 125: 287-293.

Proverbs MD 1969. Induced sterilization and control of insects. Annu Rev Entomol 14: 81-102.

Seawright JA, Bowman MC, Lofgren CS 1973. Thioaziridine chemosterilants: uptake, persistence and sterility in pupae and adults of Anopheles albimanus. J Econ Entomol 66: 305-308.

Seawright JA, Grover KK, Carlson A, Agarwal HV 1976. Studies on chemosterilization of Aedes aegypti: uptake and persistence of thiotepa in pupae and adults and the competitiveness of sterilized males. Environ Entomol 5: 849-852.

van der Wall E, Beijnen JH, Rodenhuis S 1995. High-dose chemotherapy regimens for solid tumors. Cancer Treat Rev 21: 105-132.

White GB 1966. Chemosterilization of Aedes aegypti (L.) by pupal treatment. Nature 210: 1372-1373. 